EXTENDED REPORT

\title{
Hydroxyapatite promotes superior keratocyte adhesion and proliferation in comparison with current keratoprosthesis skirt materials
}

\author{
J S Mehta, C E Futter, S R Sandeman, R G A F Faragher, K A Hing, K E Tanner, B D S Allan
}

Br J Ophthalmol 2005;89:1356-1362. doi: 10.1136/bjo.2004.064147

See end of article for authors' affiliations

\section{Correspondence to:} Mr J S Mehta, Moorfields Eye Hospital, City Road, London ECIV 2PD, UK; jodmehta@gmail.com

Accepted for publication 18 April 2005

\begin{abstract}
Aim: Published clinical series suggest the osteoodontokeratoprosthesis (OOKP) may have a lower extrusion rate than current synthetic keratoprostheses. The OOKP is anchored in the eye wall by autologous tooth. The authors' aim was to compare adhesion, proliferation, and morphology for telomerase transformed keratocytes seeded on calcium hydroxyapatite (the principal mineral constituent of tooth) and materials used in the anchoring elements of commercially available synthetic keratoprostheses. Methods: Test materials were hydroxyapatite, polytetrafluoroethylene (PTFE), polyhydroxyethyl methacrylate (HEMA), and glass (control). Cell adhesion and viability were quantified at 4 hours, 24 hours, and 1 week using a calcein-AM/EthD-1 viability/cytotoxicity assay. Focal contact expression and cytoskeletal organisation were studied at 24 hours by confocal microscopy with immunoflourescent labelling. Further studies of cell morphology were performed using light and scanning electron microscopy.

Results: Live cell counts were significantly greater on hydroxyapatite surfaces at each time point $(p<0.04)$. Dead cell counts were significantly higher for PTFE at 7 days $(p<0.002)$. $\beta_{1}$ integrin expression was highest on hydroxyapatite. Adhesion structures were well expressed in flat, spread out keratocytes on both HA and glass. Keratocytes tended to be thinner and spindle shaped on PTFE. The relatively few keratocytes visible on HEMA test surfaces were rounded and poorly adherent.

Conclusions: Keratocyte adhesion, spreading, and viability on hydroxyapatite test surfaces is superior to that seen on PTFE and HEMA. Improving the initial cell adhesion environment in the skirt element of keratoprostheses may enhance tissue integration and reduce device failure rates.
\end{abstract}

C orneal opacification is the second most common cause of world blindness affecting an estimated 10 million people. ${ }^{1}$ Although endothelial failure is the most common cause of corneal opacification in the developed world, developing world populations-in which corneal blindness is far more prevalent-are typically blind as a result of ocular surface disease leading to corneal neovascularisation and scarring. ${ }^{1}$ The prognosis for successful visual rehabilitation with conventional corneal grafting in these patients is often poor, ${ }^{2}$ and most of them have no access to allograft donor material. Exciting progress is currently being made in the development of tissue engineered alternatives to cadaveric corneal donor material. ${ }^{3}$ But, as with conventional allograft material, collagen based constructs may fail to sustain a normal corneal epithelial phenotype or stromal tissue clarity in a hostile ocular surface environment. Techniques for promoting ocular surface regeneration ${ }^{45}$ that could be used in tandem with corneal tissue replacement currently require extensive laboratory back up and are not available, even in the developed world, outside specialist centres. For the foreseeable future, the best hope of widening access to treatment for corneal blindness probably lies in the development of an improved synthetic keratoprosthesis. ${ }^{6}{ }^{7}$

Keratoprostheses incorporate a clear central optic and an annular porous surround, or skirt element, designed to provide tissue integration. ${ }^{7}$ The osteoodontokeratoprosthesis (OOKP), consisting of an annular wafer of alveolar tooth glued to a central polymethyl methacrylate optic has been implanted since the 1960s with continuing relative success. In particular, the device failure rate in association with poor tissue integration for this keratoprosthesis would appear to be significantly less than that for totally synthetic devices. ${ }^{8}$ Two totally synthetic keratoprostheses are currently available commercially: the Legeais BioKpro III (FCI Ophthalmics, Paris, France) and the AlphaCor (Argus Biomedical, Perth, Australia). Published results from clinical series of patients implanted with the Legeais BioKpro I and II suggest that up to $40 \%$ of these devices fail within one year of implantation, ${ }^{9}$ with retraction of the surface tissues over the haptic element of the device, retroprosthesis membrane formation, infection, or aqueous leakage necessitating device removal or revision of implantation. Results have not yet been published for the Legeais BioKpro III, but this device incorporates the same skirt material as earlier embodiments. Because inclusion criteria vary between case series, direct comparison of results between devices is difficult. But problems with poor tissue integration may be equally common with the AlphaCor Kpro. In a series of 40 implanted eyes followed for up to 36 months, the overall rate of surgical revision for complications associated with poor tissue integration was 30\%. ${ }^{10}$ These cases were a selected group comprised mainly of patients with a normal ocular surface, and included 14 patients who had not yet completed stage II of the implantation process in which the surface tissues are opened over the device optic. Most tissue integration problems are not clinically evident until after this surgical stage. ${ }^{11}$ In contrast to these relatively poor results for CE (Conformité Européene) marked fully synthetic Kpros, only five of 85 (5.8\%) patients implanted with the OOKP and followed for up to 16 years had clinical problems with tissue integration. ${ }^{8}$ Indications for implantation in this series included severe ocular surface disease

Abbreviations: BSA, bovine serum albumin; Ethd-1, ethidium homodimer-1; HEMA, polyhydroxyethyl methacrylate; OOKP, osteoodontokeratoprosthesis; PBS, phosphate buffered saline; PTFE, polytetrafluoroethylene; PHEMA, poly(2-hydroxyethyl methacrylate). 
secondary to ocular cicatricial pemphigoid, Stevens-Johnson syndrome, and previous chemical injury. The skirt materials used as a porous framework for tissue integration are porous polytetrafluoroethylene (PTFE) in the Legeais BioKpro III, porous polyhydroxyethyl methacrylate (HEMA) in the AlphaCor device, and autologous tooth (biological calcium hydroxyapatite) in the OOKP.

Stable tissue integration is vital for keratoprosthesis survival. Fibroblast invasion and matrix deposition has been demonstrated in vivo in preclinical studies of porous HEMA ${ }^{12}$ and PTFE $^{13}$ explanted from rabbit corneal stroma. But comparative studies to determine whether the balance between matrix synthesis and degradation may be modified by the interfacial properties of different candidate keratoprosthesis materials have not been performed in vivo. Sandeman et $a l^{14}$ have recently described a systematic approach to the evaluation of novel keratoprosthesis materials based on polyurethane based interpenetrating co-polymers. Integral to the range of assays they describe are studies of cell adhesion, viability, and morphology using a keratocyte cell strain EKl.BR. As an initial step towards exploring the cellular basis for differential extrusion rates observed clinically between these leading contemporary keratoprostheses, we performed comparative studies of cell adhesion, survival, and morphology in standard culture conditions using telomerase transformed EKI.BR keratocytes seeded on plane surfaces of PTFE, HEMA, hydroxyapatite, and glass (control).

Cellular interaction with the extracellular matrix is mediated via integrins - that is, cell surface molecules which link the extracellular matrix to the actin cytoskeleton. ${ }^{15}$ ). Integrins are composed of an $\alpha$ and a $\beta$ (18 $\alpha$ and $8 \beta$ subunits) giving a diverse range of $\alpha \beta$ combinations with their own binding specificity and signalling properties; many of the most widely expressed integrins contain the $\beta 1$ subunit. Clustering of integrins into supramolecular complexes (with cytoskeletal proteins and signalling molecules) is required to form focal contacts or adhesions. These serve as attachment sites to the underlying ECM and can also act as signalling centres regulating cell growth, survival, and gene expression. ${ }^{16}$ Further comparative work was performed to analyse the initial cellular interaction with the interfacial protein layer coating candidate keratoprosthesis materials incubated in serum enriched culture media by immunoflourescent labelling to characterise the actin cytoskeleton, focal contact, and $\beta 1$ integrin expression.

\section{MATERIALS AND METHODS \\ Cells and materials}

Telomerase transformed keratocytes were used throughout. The EKl.BR embryonic keratocyte cell strain has previously been characterised with respect to lifespan, fraction of dividing cells, and maintenance of a keratocyte phenotype in culture. ${ }^{17}$

pBABE-hTERT is a puromycin resistant amphotropic retrovirus, based on a pBABE series vector backbone, that expresses the catalytic subunit of human telomerase (hTERT), and has been described previously. ${ }^{18}$ For retroviral infections EKl.BR keratocytes were seeded at a density of $8.4 \times 10^{4}$ cells in $60 \mathrm{~mm}$ diameter standard tissue culture dishes. This seeding was performed about 24 hours before infection so that the cultures were still substantially subconfluent at the time of infection.

One hour before infection the cells were fed with fresh medium containing $8 \mu \mathrm{g} / \mathrm{ml}$ of polybrene (Sigma, St Louis, MO, USA). Two ml of supernatant from psiCRIP producer cell lines containing either pBABE-hTERT or control (pBABEpuro) retrovirus was then added to each $60 \mathrm{~mm}$ dish keeping the polybrene concentration constant at $8 \mu \mathrm{g} / \mathrm{ml}$. The cultures were then incubated for 2 hours in a tissue culture incubator at $37^{\circ} \mathrm{C}$, after which time the retroviral supernatant was diluted with fresh medium. After 24 hours the supernatant was replaced with fresh medium and cells were incubated for a further 48 hours. The cultures were then fed with selective medium (MEM containing the supplements given above together with $1.5 \mu \mathrm{g} / \mu \mathrm{l}$ puromycin). The concentration of puromycin required to provide effective selection had been determined by cytotoxicity studies undertaken in advance. At each subsequent passage total cell numbers were estimated and the population doublings estimated. EKl.BR were considered immortal after 150 population doublings.

Test materials were hydroxyapatite (Plasma Biotal, Tideswell, UK) produced by isostatic pressing hydroxyapatite powder at a nominal pressure of $50 \mathrm{MPa}$ into discs and sintering the compacts at $1250^{\circ} \mathrm{C}$, PTFE (Goodfellow Cambridge Ltd, Huntingdon, UK); and HEMA ( $34 \%$ water) (Cantor \& Nissel, Northampton, UK). Glass coverslips were used as a positive control material. The individual discs of material were stored together at room temperature before testing. All test materials were sterilised with ultraviolet irradiation for one hour before use in the experiments. All materials were used as either solid flat discs (hydroxyapatite and glass coverslips) or as flat non-porous sheet material (PTFE and HEMA).

\section{Cell adhesion, proliferation, and viability}

A viability/cytotoxicity assay using calcein-AM and ethidium homodimer-1 (EthD-1) (Molecular Probes, Eugene, OR, USA) was used to quantify viable cell growth for cells seeded at a standard concentration on each of the test materials at 4 hours, 24 hours, and 7 days. Calcein-AM is cleaved by cellular esterases present within viable cells to form a fluorescent green product which is membrane impermeable. Ethidium homodimer- 1 is a fluorescent red marker which binds to nucleic acids and only passes through the compromised membrane of non-viable cells.

$10 \mathrm{~mm}$ diameter discs of each test material were placed in the wells of a standard 12 well plate and sterilised by ultraviolet irradiation. The discs were washed three times then incubated in serum free MEM culture medium for 3 hours at $37^{\circ} \mathrm{C}$ in $5 \%$ carbon dioxide/air. Telomerase transformed EKl.BR keratocytes $\left(5 \times 10^{3}\right.$ in $2 \mathrm{ml}$ MEM containing $10 \%$ FCS) were then seeded onto each of the discs before further incubation at $37^{\circ} \mathrm{C}$ in $5 \%$ carbon dioxide/ air. At 4 hours, 24 hours, and 1 week $(n=3$ per material for each time point) culture medium was carefully removed from the wells and the cells were washed in phenol red-free medium. The discs were then incubated for 5 minutes at $37^{\circ} \mathrm{C}$ in 5\% carbon dioxide/air in phenol red-free MEM containing calcein-AM $(2 \mu \mathrm{M})$ and EthD-1 $(1 \mu \mathrm{M})$ before inverted placement in a new 12 well plate containing $1 \mathrm{ml}$ of phenol red-free MEM in each well. Cells adherent to the disc surfaces were then viewed by fluorescence microscopy. Sequential illumination at wavelengths of $500 \mathrm{~nm}$ and $625 \mathrm{~nm}$ was used to highlight calcein-AM positive (live) and EthD-1 positive (dead) cells respectively. Live/dead cell counts in five preselected fields per specimen were performed at a magnification of $\times 10$.

\section{Cell morphology}

Immunofluorescent labelling was used to examine surface expression of integrin $\left(\beta_{1}\right)$, and focal contact/adhesion formation. Organisation of the actin cytoskeleton was studied using FITC-phalloidin staining. These studies were essentially qualitative.

For immunofluorescent studies, primary labelling was with (1:100) mouse anti- $\beta_{1}$ integrin IgG (courtesy of Professor Fiona Watt, Cancer Research, UK) and (1:50) Hvin 1 mouse 


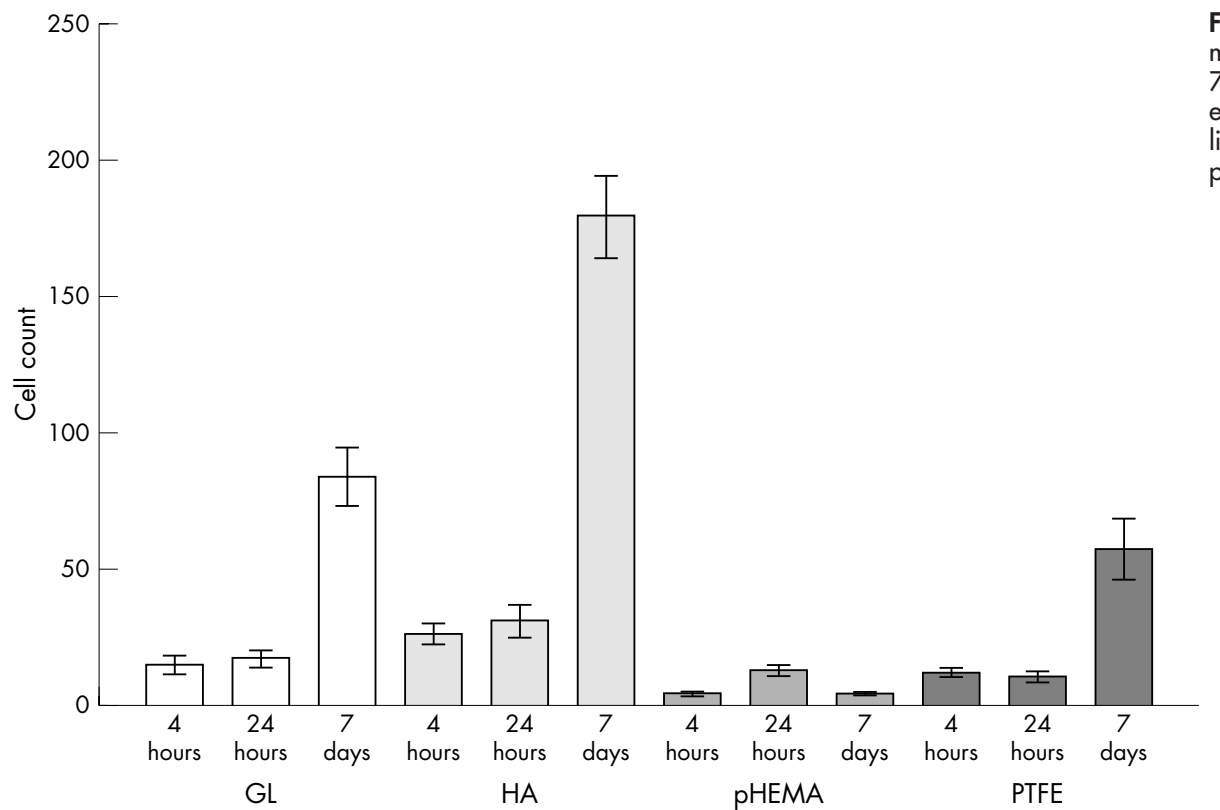

Figure 1 Live cell counts on test materials at 4 hours, 24 hours, and 7 days: error bars are $2 \times$ standard error of the mean $195 \%$ confidence limits). Cell adhesion to HEMA was too poor to permit quantification.

antivinculin IgG (Sigma, Dorset, UK). Secondary labelling was with $(1: 100)$ rhodamine conjugated donkey antimouse IgG (Jackson ImmunoResearch Laboratories Inc, West Grove, PA,USA). FITC-phalloidin (Molecular Probes, Eugene, OR, USA) was used at a concentration of 1:10.

Discs of each material $(n=4)$ were sterilised, washed, seeded with a standard innoculum of test keratocytes, and incubated for 24 hours as described above. After fixation by immersion in 3\% paraformaldehyde in phosphate buffered saline (PBS) for 30 minutes, the test materials were washed $\times 5$ in PBS and quenched $\times 2$ for 10 minutes in $15 \mathrm{mM}$ glycine diluted in PBS.

Half the samples ( $n=2$ per material) were dual labelled with antivinculin and FITC-phalloidin after cell permeabilisation with $0.1 \%$ saponin diluted in PBS for 10 minutes. Discs were blocked with $1 \%$ bovine serum albumin (BSA) in PBS for 20 minutes before incubation with primary antivinculin antibody for 2 hours, washing $\times 5$ for 5 minutes, and further incubation for 45 minutes with secondary antibody plus FITC phalloidin-all in PBS/1\% BSA/0.01\% saponin.

The remaining samples ( $\mathrm{n}=2$ per material) were blocked with BSA and labelled with anti- $\beta_{1}$ integrin primary antibody and secondary antibody exactly as above, but without prior saponin cell permeabilisation or the inclusion of saponin in subsequent buffering solutions.

After final washing, five times for 5 minutes in $1 \%$ BSA diluted in PBS (plus or minus $0.01 \%$ saponin) and twice with PBS alone, the discs were then inverted, mounted, and wax sealed onto glass slides. Subsequent imaging was performed using a Bio-Rad radiance 2000 confocal microscope (Bio-Rad Hercules, CA). We did not perform any immunofluorescent labelling studies on poly(2-hydroxyethyl methacrylate) (PHEMA) due to data obtained from the viability assay.

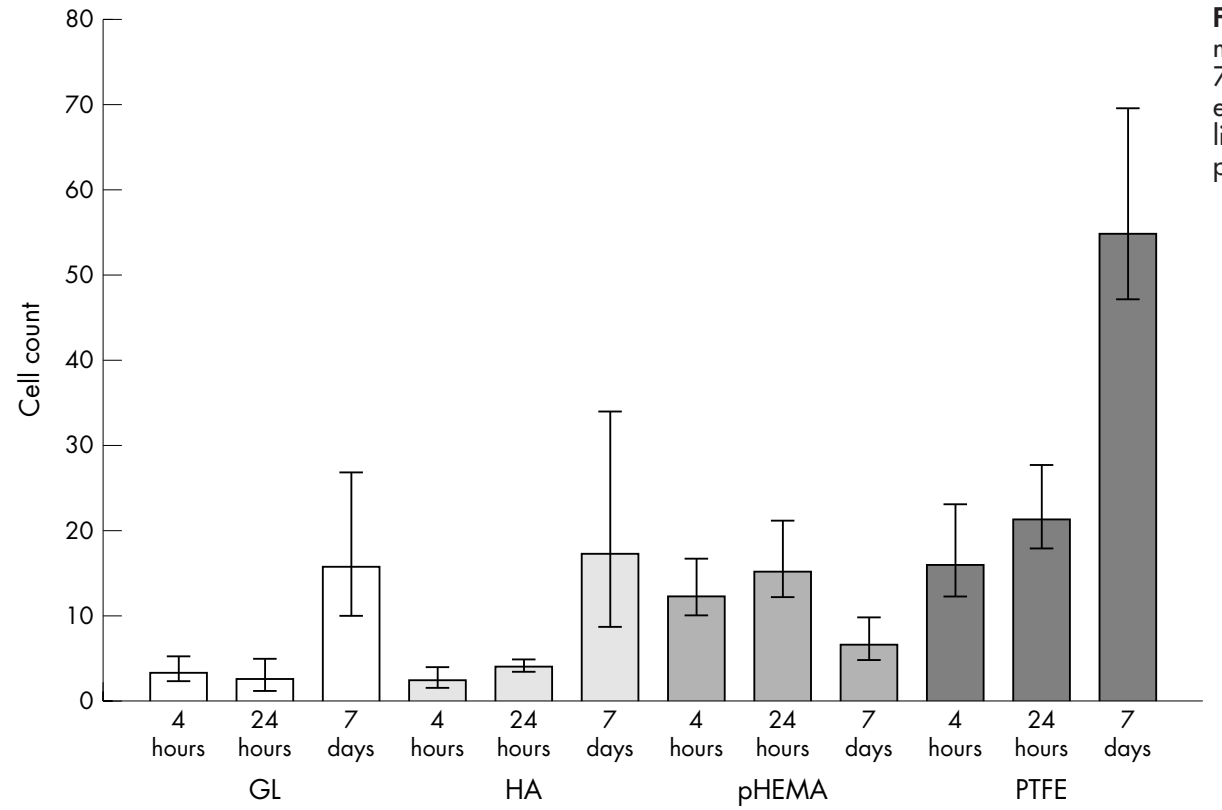

Figure 2 Dead cell counts on test materials at 4 hours, 24 hours and 7 days: error bars are $2 \times$ standard error of the mean $195 \%$ confidence limits). Cell adhesion to HEMA was too poor to permit quantification. 


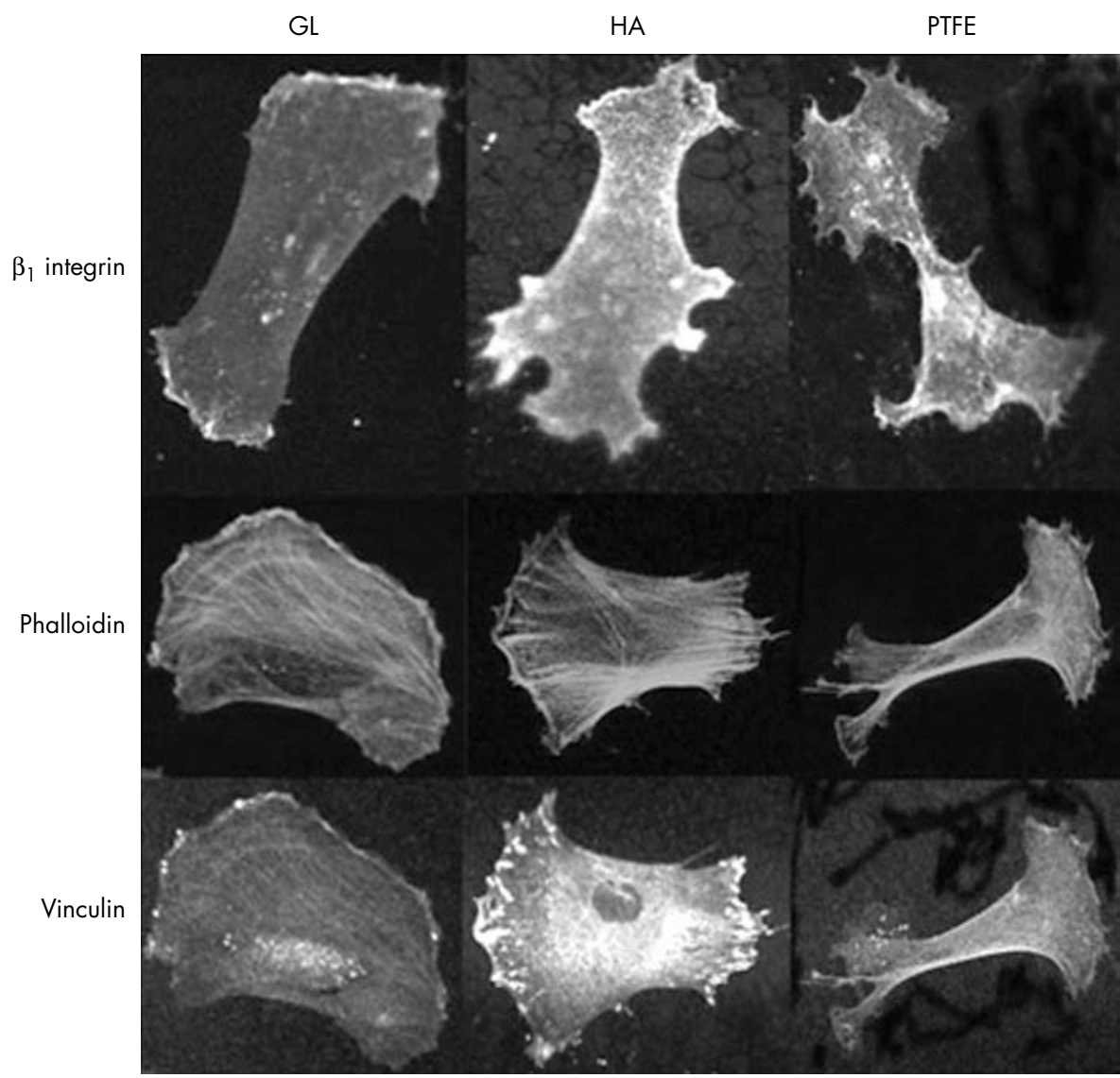

Figure $3 \quad \beta_{1}$ integrin, F-actin and vinculin distribution on test materials at 24 hours. Montage of confocal micrographs of representative cells on various test materials: $\beta_{1}$ integrin expression (immunofluorescent labeling) on glass, hydroxyapatite, and PTFE; stress fibre expression (phalloidin staining) on glass, hydroxyapatite, PTFE; and focal contact (vinculin) expression on glass, hydroxyapatite, and PTFE. Cell adhesion to HEMA surfaces was too poor to obtain confocal images.
The number of viable cells at 24 hours on this material were to few to obtain any useful images with the confocal laser.

\section{Electron microscopy}

Cells inoculated and cultured on test materials for 24 hours as described above were fixed in $2 \%$ paraformaldehyde/ $2 \%$ glutaraldehyde/0.1 M cacodylate for 30 minutes at room temperature. Specimens were then treated with $1 \%$ osmium/0.1 M cacodylate at $4^{\circ} \mathrm{C}$ for 1 hour before dehydration in ethanol, washing in hexamethyl disilazane, and drying by evaporation. After fixation, the discs were mounted on stubs using conductive silver paint and sputter coated with gold. Specimens were then examined in a JOEL 6100 (JOEL, Peabody, MA, USA) scanning electron microscope.

\section{Statistics}

SPSS version 11.5 was used to for analysis of variance to examine differences between live/dead cell counts across materials (SPSS Inc, Chicago, IL, USA). The Mann-Whitney $\mathrm{U}$ test was used to examine differences in $\beta_{1}$ integrin expression across materials. Error bars on all graphs are presented as $95 \%$ confidence limits (plus or minus $2 \times$ standard error of the mean).

\section{RESULTS}

\section{Cell adhesion, proliferation, and viablity}

Live cell counts were significantly greater for hydroxyapatite specimens at each time point than for the other test materials ( $p=0.02$ ( 4 hours), $p=0.04$ ( 24 hours), $p=0.003$ ( 1 week) (fig 1). Cell counts increased between 24 hours and 7 days on glass, hydroxyapatite, and PTFE, indicating that cells proliferated on all these materials. However, dead cell counts were significantly higher for PTFE than for other materials at 7 days $(p=0.002)$ (fig 2$)$, indicating that this material, although supporting initial adhesion, was significantly more cytotoxic than glass or hydroxyapatite.

\section{Cell morphology}

Qualitative observation of immunofluorescent labelled cells (fig 3) suggested expression of $\beta_{1}$ integrin on hydroxyapatite $>$ glass $>$ PTFE. Stress fibres (phalloidin staining) were equally prominent on hydroxyapatite and glass but less prominent on PTFE (fig 3). Focal adhesions (vinculin immunostaining) were more prominent on hydroxyapatite than on glass or PTFE (fig 3). Representative images shown in figure 4 suggest that cell spreading was greater on hydroxyapatite and glass, with cells on PTFE adopting a more spindle shaped, elongated form.

Light microscopic observation during the cell adhesion, proliferation, and viability assays suggested more rapid cell spreading on hydroxyapatite than the other test materials (fig 4). After 4 hours cells were round on the HEMA, a mixture of round and spreading on glass and PTFE, and mainly spreading on hydroxyapatite. At 24 hours cells on HEMA were still mainly round with few cells spreading out; but on glass, hydroxyapatite, and PTFE the cells were mainly spread out. At 1 week there was a mass of cells on the glass, hydroxyapatite, and PTFE; however the scant cell deposits on HEMA were still round and poorly adherent. Scanning electron microscopy, at 24 hours, was consistent with these observations (fig 5). On hydroxyapatite cells were spread so thinly that the surface texture of the test material was clearly visible through flattened lamellipodia. Lamellipodia of cells on glass were not as thin as those on hydroxyapatite, but were, nevertheless, well spread and showed more filopodia than cells cultured on hydroxyapatite. Cells cultured on PTFE were much less well spread than those on HA or glass, 


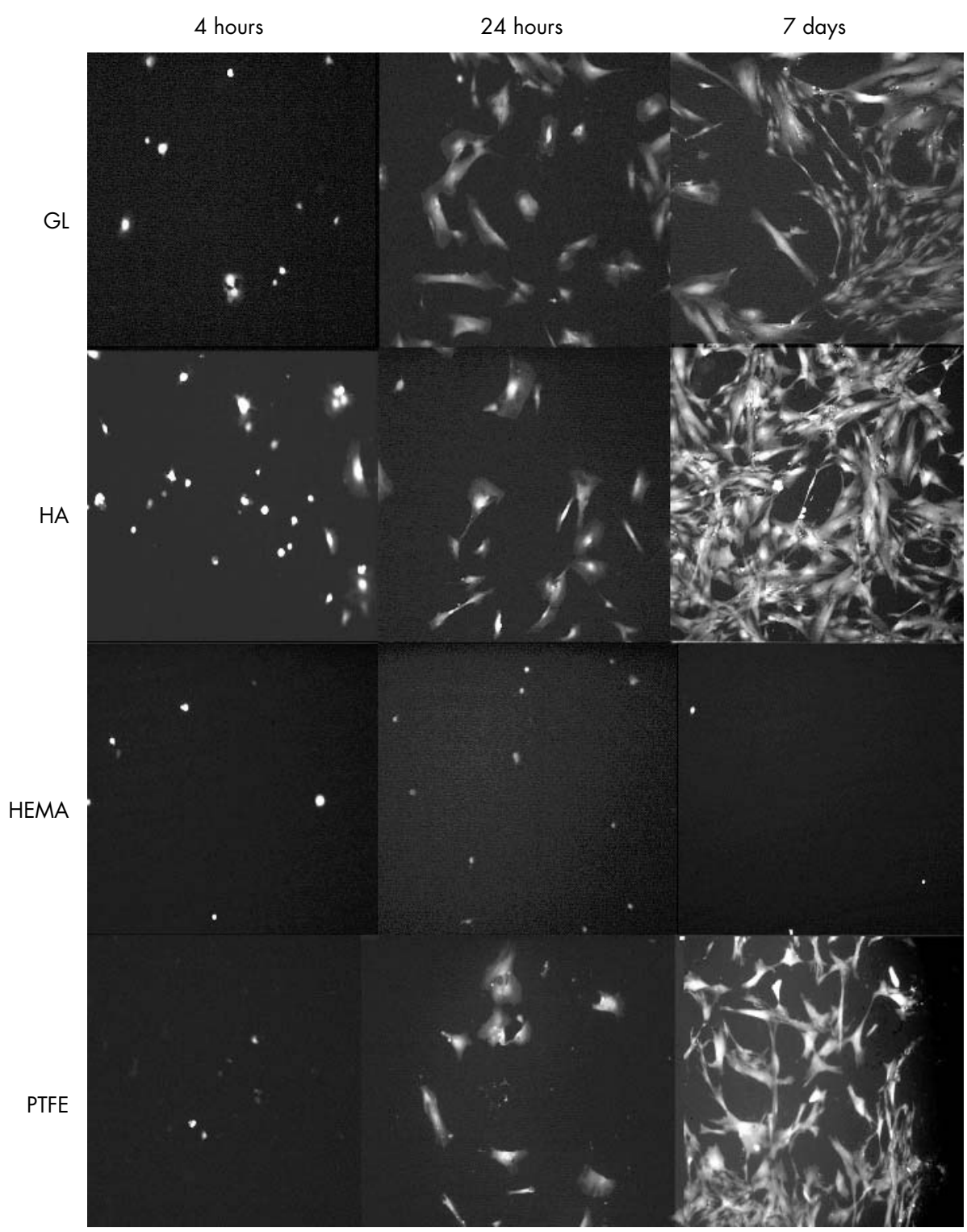

Figure 4 Light microscopy showing cell morphology on test materials. Montage of calcein-AM stained keratocytes illustrating cell morphology at different time points ( 4 hours, 24 hours, 7 days) on glass (GL), hydroxyapatite (HA), HEMA, and PTFE.

produced numerous long extensions, and were frequently blebbing.

\section{DISCUSSION}

These basic studies of cell adhesion, proliferation, and survival suggest that hydroxyapatite is superior, as an initial adhesion environment for keratocytes, to inert plastics used as a porous tissue integration framework in contemporary CE marked keratoprosthesies. Cell adhesion to pHEMA was poor and cell death was prominent on PTFE.

We used telomerase transformed keratocytes in an effort to standardise our experimentation through the use of a cell line with a near normal phenotype. Transduction of cells to express the catalytic subunit of telomerase permits them to retain their telomeres and divide indefinitely without global alterations of gene expression produced by earlier methods of cellular immortalisation. ${ }^{19}$ The use of telomerase transformed cells is increasing in ophthalmic research ${ }^{20} 21$ and should help to enhance both the reproducibility and biological validity of experimental results.
Cells were derived from the previously characterised EK1BRl human keratocyte strain. ${ }^{17}$ In the presence of serum, and specifically TGF- $\beta$, keratocytes develop a myofibroblast phenotype. ${ }^{22}$ Clinically, keratoprosthesis skirts may be colonised by fibroblasts derived from sclera, or the overlying mucous membrane flap. Although we have not performed comparative studies, these cells have similar adhesion structures, and it is unlikely that their behaviour would differ significantly from keratocyte derived myofibroblasts.

The study was restricted to plane surfaces. Cells may behave differently in colonising a 3D porous keratoprosthesis skirt. ${ }^{7}$ Surface texture also exerts an important influence on cell behaviour, ${ }^{23}$ and this was uncontrolled. Despite these limitations, the fit between our principal findings and the relatively good tissue integration observed clinically for the $\mathrm{OOKP}^{8}$ is striking.

Our failure to observe cell adhesion on unmodified HEMA surfaces is consistent with earlier published results. ${ }^{24}{ }^{25}$ Peluso et al demonstrated human embryonic lung fibroblasts plated on HEMA fail to spread after 24 hours and remain spherical in shape, while those plated on modified HEMA 


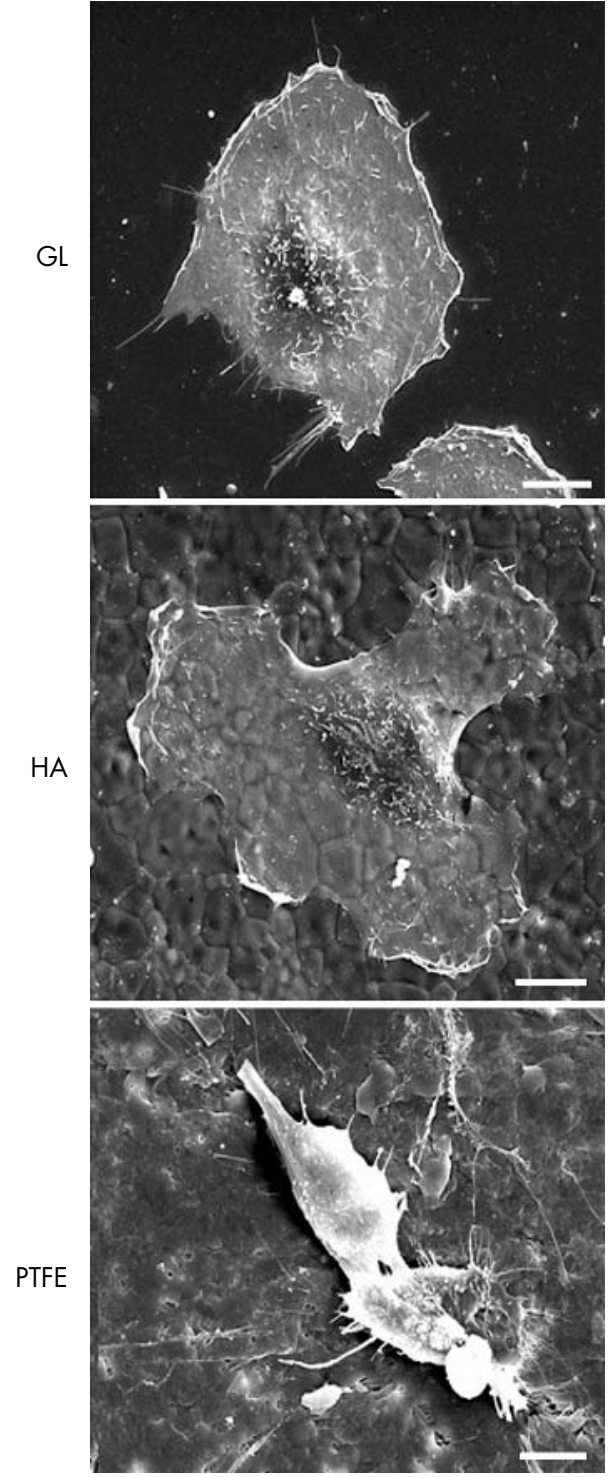

Figure 5 Scanning electron microscopy showing cell morphology on test materials. Montage of scanning electron micrographs showing keratocyte morphology at 24 hours on glass, HA, and PTFE. Bar $=10 \mu \mathrm{m}$.

demonstrated good cell attachment and spreading. ${ }^{24}$ Sandeman et al also observed poor cellular attachment to unmodified HEMA at 24 hours using the EKI.BR cell line but showed spreading of cells if the HEMA was modified with 15\% phenoxyethyl methacrylate (PEM) or of $20 \%$ 2-(dimethylamino)ethyl methacrylate (DEM). ${ }^{25}$ Even at 1 week, little evidence of cellular attachment to unmodified HEMA was observed. Although inferior to glass or hydroxyapatite, cell adhesion to PTFE surfaces was relatively good, but characterised by high levels of cell death.

Failure of cell adhesion to pHEMA and relatively high levels of cell death observed for PTFE may adversely influence tissue integration for the AlphaCor and Legeais BioKpro 111 respectively. Both keratoprostheses have a published track record of development which includes histological studies of explants from intralamellar corneal implantation. ${ }^{26}{ }^{27}$ These studies, and others, ${ }^{7}$ demonstrate cellular ingrowth, collagen and matrix deposition, but do not demonstrate matrix stability. Future studies of gene expression focussing on matrix synthesis and degradation may reveal important differences among fibroblasts colonising different candidate keratoprosthesis materials.

Hydroxyapatite is similar to the principal mineral constituent of bone and tooth, and is thought to promote integration between hard and soft tissues by adsorbing extracellular adhesion molecules in a 3D configuration that preferentially exposes a high density of integrin binding sites. ${ }^{28}$ We observed qualitatively greater $\beta 1$ integrin expression in cells adherent to hydroxyapatite versus other test materials. The strength of cell adhesion to a surface is determined not only by the numbers of surface integrin receptors, but also by their organisation. Integrins are organised into oligomeric complexes, termed focal adhesions/complexes, which link the extracellular matrix with the actin cytoskeleton. Increased numbers of focal adhesions observed in cells adherent to hydroxyapatite may account for the increased adhesion to hydroxyapatite seen in live/dead cell counts and the close apposition of keratocytes to the hydroxyapatite shown by standard error of the mean.

A range of bioactive ceramic materials has been developed to encourage bonding with soft tissues through an interfacial layer of hydroxyapatite. Existing applications include ossicular replacement, periodontal bone regeneration, and orbital floor repair. ${ }^{28}$ Porous hydroxyapatite coral implants are widely used in ophthalmology as post enucleation ball implants. ${ }^{29}$ A keratoprosthesis with a coral skirt element has also been described. ${ }^{30}$ However, a rigid hydroxyapatite skirt could potentially lead to problems with device fracture, and stress concentrations at the skirt/sclera interface could encourage tissue breakdown. Although bioactive ceramics are available as resorbable gels and metallic surface coatings, coating a flexible polymer is less easy. The ideal keratoprosthesis would have a modulus of elasticity similar to the eye wall. ${ }^{7}$ Flexible polymer surfaces incorporating integrin adhesion ligands such as the RGD or YIGSR motif have recently been developed..$^{31}$ A logical future extension of our study would encompass these materials.

The selection of inert plastic materials such as PTFE and HEMA as keratoprosthesis skirt materials has been based on their record of safe implantation rather than any special merit as an interfacial surface to promote normal tissue differentiation. ${ }^{72}$ We have demonstrated that hydroxyapatite is a superior starting adhesion environment for keratocytes. This may help to explain relatively low extrusion rates observed clinically for the OOKP, ${ }^{8}$ and suggests that material selection for the next generation of keratoprostheses should move away from inert plastics towards materials designed to promote normal adhesion and differentiation in contacting cells.

\section{ACKNOWLEDGEMENTS}

The hTERT vector supplied under a material transfer agreement with Geron Corporation (Geron Corporation, Menlo Park, CA). The authors acknowledge funding from the Special Trustees of Moorfields Eye Hospital and the Sir Jules Thorn Charitable Trust.

\section{Authors' affiliations}

J S Mehta, B D S Allan, Cornea \& External Disease Service, Moorfields Eye Hospital, London, UK

J S Mehta, C E Futter, B D S Allan, Division of Cell Biology, Institute of Ophthalmology, London, UK

S R Sandeman, R G A F Faragher, University of Brighton, School of Pharmacy and Biomolecular Science, University of Brighton, Brighton, UK

K A Hing, K E Tanner, IRC in Biomedical Materials, Queen Mary University of London, London, UK

Competing interests: none declared

This paper was a presentation at the Association for Research in Vision and Ophthalmology, April 2004, Fort Lauderdale, FL, USA. 


\section{REFERENCES}

1 World Health Organisation. The World Health Report 1998: life in the 21 st century. Geneva: WHO, 1998:47.

2 Williams KA, Roder D, Esterman A, et al. Factors predictive of corneal graft survival: report from the Australian Corneal Graft Registry. Ophthalmology 1992;99:403-14

3 Li F, Carlsson D, Lohmann C, et al. Cellular and nerve regeneration within a biosynthetic extracellular matrix for corneal transplantation. Proc Nat Acad Sci 2003; 100:15346-51

4 Pellegrini G, Traverso CE, Franzi AT, et al. Long-term restoration of damaged corneal surfaces with autologous cultivated corneal epithelium. Lancet 1997:349:990-3.

5 Grueterich M, Espana EM, Tseng SC. Ex vivo expansion of limbal stem cells: amniotic membrane serving as a stem cell niche. Surv Ophthalmol 2003;48:631-46

6 Allan BDS. Artificial corneas. BMJ 1999;318:821-2.

7 Hicks CR, Fitton HF, Chirila TV, et al. Keratoprostheses: advancing toward a true artificial cornea. Surv Ophthalmol 1997;42:175-89.

8 Marchi V, Riici R, Pecorella l, et al. Osteo-odonto-keratoprosthesis. Description of the surgical technique with results in 85 patients. Cornea 192, 13:125-30.

9 Legeais J-M, Renard G. A second generation of artificial cornea (Biokpro II). Biomaterials 1998;19:1517-22.

10 Hicks CR, Crawford GJ, Lou X, et al. Corneal replacement using a synthetic hydrogel cornea Alphacor device preliminary outcomes and complications. Eye 2003; 17:385-92

11 Dohlman CH, Doane MG. Some factors influencing outcome after keratoprosthesis surgery. Cornea 1994;13:214-18.

12 Chirila TV, Constable IJ, Crawford GJ, et al. Poly(2-hydroxy-ethyl methacrylate) sponges as implant materials: in vivo and in vitro evaluation of cellular invasion. Biomaterials 1993;14:26-38.

13 Legeais JM, Rossi C, Renard G, et al. A new fluorocarbon for keratoprosthesis. Cornea 1992;11:538-45.

14 Sandeman SR, Llloyd AW, Tighe BJ, et al. A model for the preliminary screening potential keratoprosthetic biomaterials. Biomaterials 2003;24:4729-39.

15 Van der Flier A, Sonnenberg A. Functions and interactions of integrins. Cell Tissue Res 2001;305:285-98.

16 Giancotti FG, Ruoslahti E. Integrin signalling. Science 1999;285:1028-32.
17 Dropcova S, Denyer S, Lloyd A, et al. A standard strain of human ocular keratocytes. Ophthalmic Res 1999;31:33-41.

18 Wyllie FS, Jones CJ, Skinner JW, et al. Telomerase prevents accelerated cell ageing of Werner syndrome fibroblasts. Nat Genet 2000 24:16-17.

19 Jiang XR, Jimenez G, Chang E, et al. Telomerase expression in somatic cells does not induce changes associated with a transformed phenotype. Nature Genet 1999;21:111-14

20 Ranbhatla L, Chiu CP, Glickman RP, et al. In vitro differentiation capacity of telomerase immortalised human RPE cells. Invest Ophth Vis Sci 2002;43: 1622-30

21 Gipson IK, Spurr Michaud S, Argueso P, et al. Mucin gene expression in immortalised human corneal-limbal and conjunctival epithelial cell lines. Invest Ophth Vis Sci 2003;44:2496-506.

22 Jester J, Ho-Chang T. Modulation of cultured corneal keratocyte phenotype by growth factors/cytokines controls in vitro contractility of extracellular matrix contraction. Exp Eye Res 2003;77:581-92.

23 Curtis AS, Wilkinson CD. Reactions of cells to topography. J Biomater Sci Polym Ed 1998;9:1313-29.

24 Peluso G, Petillo O, Anderson JM, et al. The differential effects of poly(2hydroxyethylmethacrylate) and poly(2-hydroxyethyl methacrylate) / poly(caprolactone) polymers on cell proliferation and collagen synthesis by human ling fibroblasts. J Biomed Mater Res 1997;34:327-36.

25 Sandeman SR, Faragher RGA, Allen MCA, et al. Novel materials to enhance keratoprosthesis integration. $\mathrm{Br} J$ Ophthalmol 2000;84:640-4.

26 Chirila TV, Constable IJ, Crawford GJ, et al. Poly (2-hydroxyethyl methacrylate) sponges as implant materials: in vivo and in vitro evaluation of cellular invasion. Biomaterials 1993;14:26-38.

27 Legeais JM, Rossi G, Renard G, et al. A new fluorocarbon for keratoprosthesis. Cornea 1992;11:538-45.

28 Allan BDS. Closer to nature: new biomaterials and tissue engineering in ophthalmology. Br J Ophthalmol 1999;83:1235-40.

29 Ashworth JL, Rhatigan M, Sampath R, et al. The hydroxyapatite orbital implant: a prospective study. Eye 1996;10:29-37.

30 Leon CR, Baraquer JL Jr, Barraquer JL Sr. Coralline hydroxyapatite keratoprosthesis in rabbits. J Refract Surg 1997;13:74-8.

31 Dee KC, Anderson TT, Bizios R. Design and function of novel osteoblast adhesive peptides for chemical modification of biomaterials. J Biomed Mater Res 1998;40:371-2. 